\title{
Los desafíos de las ciudades vulnerables. Construcción de ciudad y construcción del riesgo en La Paz y El Alto
}

Les défis des villes vulnérables. Construction de la ville et construction du risque à La Paz et El Alto

The challenges of vulnerable cities: construction of the city and construction of risk in La Paz and El Alto

\section{Patricia Urquieta C.}

\section{OpenEdition}

\section{Journals}

Edición electrónica

URL: http://journals.openedition.org/bifea/5906

DOI: 10.4000/bifea.5906

ISSN: 2076-5827

Editor

Institut Français d'Études Andines

Edición impresa

Fecha de publicación: 1 diciembre 2014

Paginación: 445-462

ISSN: 0303-7495

Referencia electrónica

Patricia Urquieta C., " Los desafíos de las ciudades vulnerables. Construcción de ciudad y

construcción del riesgo en La Paz y El Alto », Bulletin de l'Institut français d'études andines [En línea], 43

(3) | 2014, Publicado el 08 diciembre 2014, consultado el 05 noviembre 2020. URL : http:// journals.openedition.org/bifea/5906; DOI : https://doi.org/10.4000/bifea.5906

\section{(c) (†) $\odot$}

Les contenus du Bulletin de l'Institut français d'études andines sont mis à disposition selon les termes de la licence Creative Commons Attribution - Pas d'Utilisation Commerciale - Pas de Modification 4.0 International. 


\title{
Los desafíos de las ciudades vulnerables. Construcción de ciudad y construcción del riesgo en La Paz y El Alto
}

\section{Patricia Urquieta C.}

... la ciudad es el mundo que el hombre ha creado, también constituye el mundo donde está condenado a vivir en lo sucesivo. Así pues, indirectamente y sin tener plena conciencia de la naturaleza de su obra,

al crear la ciudad, el hombre se recrea a sí mismo. Robert Park, La ciudad y otros ensayos de ecología urbana (1999)

\begin{abstract}
Resumen
En este artículo se plantea la necesidad de un giro metodológico para estudiar los riesgos en las ciudades desplazando la mirada más allá del binomio amenaza-vulnerabilidad hacia el funcionamiento de la sociedad. Se intenta aportar a la discusión de lo urbano desde el significado de las ciudades y en la perspectiva del desarrollo social. Al comprender el espacio como una construcción social es posible reconocer la complejidad de factores y formas de la ocupación del suelo, esto es, incluir la necesidad como un determinante de la formación de las ciudades, junto al mercado y a las acciones o falta de acciones del Estado. Se sostiene que la respuesta a esta realidad debe ser integral y solidaria: la construcción de la ciudad, lo mismo que la gestión del riesgo, deben verse como una corresponsabilidad entre Estado y sociedad.
\end{abstract}

Palabras clave: urbanización, espacio social, vulnerabilidad, corresponsabilidad, La Paz, El Alto

Máster en Estudios Culturales por la Universidad Andina Simón Bolívar de Quito y candidata a doctora en Ciencias del Desarrollo. Coordinadora de la Unidad de Estudios Urbanos y docente investigadora del Posgrado en Ciencias del Desarrollo de la Universidad Mayor de San Andrés (CIDES-UMSA) de La Paz, Bolivia. 


\title{
Les défis des villes vulnérables. Construction de la ville et construction du risque à La Paz et El Alto
}

\begin{abstract}
Résumé
Cet article propose un changement de cap méthodologique pour étudier la dynamique des villes en déplaçant le regard au-delà du binôme menace-vulnérabilité vers le fonctionnement de la société. II vise à contribuer à la discussion sur «l'urbain » comme un concept qui comprend le sens des villes, et dans la perspective du développement social. En analysant l'espace comme une construction sociale il est possible de reconnaître la complexité des facteurs et des formes d'utilisation des terrains. C'est-àdire, inclure le besoin comme un déterminant de la formation des villes, avec le marché et les actions ou l'absence d'actions de l'État. Nous soutenons que la réponse à cette réalité doit être globale et solidaire : la construction de la ville, ainsi que la gestion des risques, doivent être considérés comme une responsabilité partagée entre l'État et la société.
\end{abstract}

Mots clés : urbanisation, espace social, vulnérabilité, responsabilité partagée, La Paz, El Alto

\section{The challenges of vulnerable cities: construction of the city and construction of risk in La Paz and El Alto}

\begin{abstract}
This paper shows the need of a methodological shift in urban studies from the examination of threat and vulnerability dynamics formulas towards a more complete comprehension of how urban systems operate. It contributes to discussions on the meanings of urban systems and their role in social development. Understanding urban spaces as social constructions leads to the recognition of a complex set of factors and parallel forms of land use, including need as a determinant in the formation of cities, the presence of diverse markets and the actions and lack of actions of the State. It is argued that solutions to this reality must be comprehensive and include elements of solidarity and that the construction of cities and risk management within urban settings should be seen as a responsibility shared between State and society.
\end{abstract}

Keywords: urbanization, social space, vulnerability, shared responsibility, La Paz, El Alto

\section{INTRODUCCIÓN}

Es conocido que muchas ciudades latinoamericanas se construyen sin planificación alguna, o que esta aparece tardíamente para plantear normas que no siempre son atendidas por la sociedad, lo que a su vez produce mayor caos y empeora las condiciones ya precarias. Parecería que el precio para acceder a la ciudad —del desarrollo urbano, en general- es la convivencia con el riesgo. El aumento de la población en las ciudades, concentrada en escaso territorio urbano y siguiendo un patrón centrífugo, ha creado existencias en peligro, dada la suma de vulnerabilidades estructurales, de largo plazo, «de fondo», momentáneas, históricas y «circunstanciales».

Una preocupación recurrente en la planificación de la ciudad y en materia de prevención de riesgos y desastres es saber si la sociedad tiene el conocimiento e 
información oportuno y disponible sobre la amenaza que suponen los fenómenos naturales, socio-naturales, los denominados antrópico-maliciosos y los de origen tecnológico. También se busca saber en qué medida la población está expuesta a ellos y si esta sabe cuan vulnerable es su situación.

Desde la perspectiva de la población y de las instituciones con dominio técnico sobre el tema, la preocupación es saber si la institución pública — principalmente a nivel de los gobiernos locales - tiene la capacidad de responder a la demanda de acciones de prevención, estimación/mitigación, preparación, alerta, respuesta y reconstrucción.

Por otra parte, el uso y ocupación del suelo y las condiciones de la vivienda en América Latina son factores que «pesan» cuando se piensa en los escenarios de desastre y se proyecta acciones y políticas urbanas de prevención. Posiblemente se deba a ello el reclamo de mayor investigación en nuestra región alrededor de las dimensiones sociales de la gestión del riesgo.

Es en este sentido que se plantea la necesidad de operar un giro metodológico para estudiar la dinámica de las ciudades. Aunque la clásica definición del riesgo se construyó alrededor del binomio amenaza-vulnerabilidad y la gestión del riesgo priorizó la amenaza en su enfoque de intervención, hoy se debate y aplica nuevos planteamientos que buscan no concentrarse exclusivamente en la amenaza sino en el funcionamiento de la sociedad para responder mejor a las necesidades tanto de los gestores como de las poblaciones (D'Ercole \& Metzger, in Hardy, 2009a).

En el presente artículo se intenta aportar a la discusión sobre la importancia de comprender lo urbano desde el significado de las ciudades y en la perspectiva del desarrollo social. Se sostiene que el modelo de urbanización (de desarrollo) que se aplica en Bolivia, como en muchas ciudades latinoamericanas, no solo produce inequidad y desigualdad, segregación y autosegregación, sino también riesgo y vulnerabilidad.

El debate alrededor de los estudios urbanos en la región planteaba, a fines de los años 1980, la posibilidad de hablar de una urbanización andina con particularidades propias, relacionadas a su realidad pluricultural y pluriétnica (Carrión, 1988; Sandóval, 1988; Sandoval \& Sostres, 1989; Saravia \& Sandoval, 1991) y si esta podría «aportar en la construcción colectiva de una nueva interpretación de los procesos urbanos latinoamericanos» desde un enfoque más localizado pero entendiendo lo local como un ámbito micro de la sociedad global (Carrión, 1988: 13-15).

El espacio habitable en las ciudades andinas de Bolivia — para la amplia mayoría de sus pobladores - es escaso y el suelo no apto para la construcción de viviendas. A esta precariedad del suelo se suma el hecho de que la mayor parte de los asentamientos urbanos son autoconstruidos (el 70\% de las viviendas en La Paz y el 80\% en El Alto) (Fopevi, 2009) e informales. Solo más tarde el proceso de consolidación se irá alcanzando: conformada la junta vecinal se «luchará» para, primero, lograr la inscripción formal de la vivienda y, luego, poco a poco se «conseguirán» los servicios básicos. Así, al construir la vivienda se construye vulnerabilidad y, al formalizar la autoconstrucción, esta se consolida. 
Comprender el espacio como una construcción social conduce a reconocer la complejidad de factores y formas de la ocupación del suelo en los procesos de urbanización de las ciudades. Del mismo modo, incluir la necesidad como un determinante de la formación de las ciudades, junto al mercado y a las acciones o falta de acciones del Estado, podría ayudar a desatorar algunos nudos en la investigación urbana.

Planteamos aquí que la respuesta a esta realidad debe ser integral y solidaria: la construcción de la ciudad, lo mismo que la gestión del riesgo, es una corresponsabilidad entre el Estado y la sociedad.

\section{LA CONSTRUCCIÓN SOCIAL DEL ESPACIO}

Las diferentes acepciones que conocemos del espacio, desde la perspectiva de las ciencias sociales, pueden ser rastreadas a partir de tres grandes visiones filosóficas, explican Hiernaux \& Lindón (1996): a) la que distingue el espacio como continente o receptáculo, b) aquella que entiende el espacio como reflejo y c) la que ve el espacio como una instancia o dimensión de la totalidad social.

En el primer caso, el espacio es entendido como un contenedor de objetos, sin la capacidad de incidir sobre los elementos que en él se manifiestan; en el segundo caso también se trata de una noción pasiva en la que el espacio es concebido como un espejo de las relaciones sociales, es decir, que todo cambio en la sociedad es reflejado inmediatamente en él; la tercera visión, que entiende el espacio como estructura o totalidad social, surge de distintos aportes disciplinarios — desarrollada principalmente por la geografía crítica- - « «procede de autores latinoamericanos identificados con la problemática regional y territorial, como Rofman o Coraggio». Desde este punto de vista «el espacio es una realidad objetiva, más allá de las particulares percepciones que de él puedan tener los individuos (...) es función de una sociedad al mismo tiempo que existe en sí mismo, y en este sentido se impone a la sociedad» (Hiernaux \& Lindón, 1996: 92).

Nutrida de estas reflexiones epistemológicas, la geografía ha desarrollado diversas concepciones del espacio de acuerdo a su contexto de influencias. En la década de los años 1960, marcada por el análisis urbanístico y la antropología urbana y más tarde en la década de 1970, bajo la influencia de la psicología social, surge la noción de que en la representación del espacio influyen tanto la experiencia individual como las formas de socialización, por consiguiente, la cultura ((Hiernaux \& Lindón, 1996: 97). Más aún, la geografía crítica latinoamericana liderada por Milton Santos considera al espacio «como un conjunto de relaciones que se desarrollan a través de funciones y de formas que representan una historia escrita por procesos del pasado y del presente» (Hiernaux \& Lindón, 1996: 103).

Así, el espacio, objeto de la geografía, es un espacio social, dirá Ortega Valcárcel citado en Villafañez (2011), producto de la acción humana; no es un objeto dado ni preexistente sino que se produce socialmente, históricamente. 
El espacio como producto social es un objeto complejo y polifacético: es lo que materialmente la sociedad crea y recrea, con una entidad física definida; es una representación social y es un proyecto en el que operan individuos, grupos sociales, instituciones, relaciones sociales, con sus propias representaciones y proyectos. El espacio se nos ofrece, además, a través de un discurso socialmente construido, que mediatiza al tiempo que vehicula nuestra representación y nuestras prácticas sociales (Villafañez, 2011: 150, énfasis añadido).

\section{LA PRODUCCIÓN DEL ESPACIO Y EL ESTUDIO DE LO URBANO}

Testigo de los cambios evidentes en el proceso de urbanización en el mundo, a principios de la década de 1970, Lefebvre (1974) anunciaba el nacimiento de un nuevo concepto: el de la producción del espacio. Intentando describir una nueva relación de la sociedad con el espacio. Se refirió a los espacios urbanos como los puntos fuertes de confluencia de los flujos económicos, para explicar cómo el capitalismo se apoya, no solamente sobre las empresas y el mercado, sino sobre el espacio. De acuerdo a su hipótesis:

Es el espacio y por el espacio donde se produce la reproducción de las relaciones de producción capitalista. El espacio deviene cada vez más un espacio instrumental (...) un espacio abstracto, como lo es el espacio del hormigón, por ejemplo (Lefebvre, 1974: 223).

A fines de la década de 1980, Carrión (1988) escribió que era posible plantearse la existencia de una urbanización andina, es decir, que hubiera existido un proceso de formación de las ciudades latinoamericanas del Ande con particularidades propias; que su conocimiento y caracterización podría aportar a las discusiones que se desarrollaban sobre lo urbano en ese momento. Era el tiempo en que se reconocía que las teorías de la urbanización existentes habían mostrado sus limitaciones para comprender las complejidades de estas «otras ciudades» de la región y que exigían métodos más locales, con énfasis en estudios de caso que den mayor importancia al particularismo como fuente de conocimiento en lugar de las generalizaciones.

Se intentaba ir más allá de las contradicciones sociales (la desigualdad e inequidad entendidas de manera general) como la principal perspectiva de aquellas teorías urbanas.

La realidad pluricultural y pluriétnica, propia del mundo andino, [permitiría] — de acuerdo al vaticinio de Carrión- formular aportes alrededor de los llamados economicismos, culturalismos, multidimensionalidades de las definiciones sobre la problemática urbana (Carrión, 1988: 13-23).

Para Mazurek (2006) lo urbano es la cristalización de lo social y lo espacial.

La ciudad, lo urbano, su crecimiento son un fenómeno complejo donde lo simbólico, lo cultural, lo social, lo geográfico, lo económico, etc. se entrelazan con la historia (Mazurek, 2006: 28). 
La perspectiva histórica, precisamente -que privilegia el estudio de la estructuración urbana, la conformación espacial, la dinámica de crecimiento de las ciudades, etc.- dará las pautas para comprender cómo se producen las desigualdades basadas en la ocupación del suelo.

Aunque la formación urbana en cada caso refleja un modelo cultural, también es posible plantear una estructuración urbana de rasgos universales, «un modelo centro-periferia de tipo exponencial negativo» generador de segregación social y espacial. Efectivamente, si bien las ciudades miradas desde lejos simbolizan riqueza y progreso son, al mismo tiempo, la fuente generadora de desigualdad 1. Citando a Baron, pero probablemente mirando las ciudades andinas bolivianas, Mazurek dirá que la construcción de redes socioeconómicas alternativas y modalidades propias de territorialidad forman parte de la reacción o estrategias de respuesta a los procesos de exclusión que se dan en las ciudades en el nuevo marco de modernidad (2006: 27-33).

\section{LO URBANO EN BOLIVIA}

Como parte de los países latinoamericanos de urbanización tardía ${ }^{2}$ y debido al débil desarrollo de sus fuerzas productivas, en Bolivia también se experimentó la priorización de los temas rurales en la investigación social (Urquieta, 2010). De acuerdo a Sandoval (1988), la indagación más constante sobre lo urbano fue realizada por arquitectos urbanistas. Hasta la década de 1990, las otras ramas científicas abordaron la problemática urbana influidas «más por las exigencias del crecimiento de los problemas y necesidades de consumo colectivo de la población urbana en expansión que por un interés por comprender la organización territorial y las relaciones sociales que allí se desarrollan» (Sandoval, 1988: 61)3.

Sin embargo, en los últimos años, aunque todavía dispersa, la investigación sobre la temática urbana es abundante y se realiza desde las distintas disciplinas de las

1 Según Harvey (2008) la urbanización desempeña un papel importante para absorber el producto excedente del capitalismo, por eso las grandes revoluciones urbanas se han dado cada vez que el capital necesitaba oxígeno o debía estabilizar una situación de crisis. La transformación urbana basada en estas razones provoca una reestructuración de la ciudad en función de las clases sociales, privilegia a unos y margina a los otros. Por eso, dado que el proceso urbano es el canal esencial de uso del excedente, instituir una gestión democrática en este proceso es lo que constituye el derecho a la ciudad. El espacio como derecho.

2 Junto a Ecuador, Paraguay y algunos países de Centro América. Bolivia se convirtió en un país predominantemente urbano a fines de los años 1980, casi tres décadas después de Argentina, Chile y Uruguay.

3 Carrión sostiene que la investigación urbana en el área andina es reciente no solamente por su tardío proceso de urbanización, sino también porque las ciencias sociales dieron a las ciudades andinas una jerarquía de segundo nivel, las ubicaron en un lugar secundario a la hora de analizar los procesos de formación social, por considerarlas sociedades agrarias, atrasadas, tradicionales, más rurales que urbanas. «La misma teoría y acción política impidieron entender estas formaciones porque las concebían como un epifenómeno resultante de contradicciones más importantes (primarias) y por lo tanto no fundamentales; o como problema local que no concierne a la sociedad nacional» (Carrión,1988: 18). 
ciencias sociales, en muchos casos, con enfoque interdisciplinario. Estos estudios abordan no solamente lo concerniente al crecimiento físico de las ciudades y las complejidades que de ello emanan para la planificación y la gobernabilidad, sino que vinculan aquello a su dinámica interna, a su dimensión social y cultural y a las diversas formas de apropiación del espacio.

La realidad que representa hoy el incesante proceso de urbanización y el crecimiento poblacional y espacial de las ciudades bolivianas, tal como lo demuestra el Censo Nacional de Población y Vivienda 2012, no solo provee una riqueza de información para la investigación sino también exige mayor atención de las ciencias sociales.

\section{EL PROCESO DE URBANIZACIÓN DE LA CIUDAD DE LA PAZ}

Excepto algunos intentos de planificación para el ordenamiento de las ciudades, desde una perspectiva institucional y de gestión, en Bolivia lo urbano ha sido un tema sistemáticamente postergado: ni el urbanismo ni el desarrollo urbano fueron una prioridad para el Estado y hasta hoy la ausencia de políticas públicas al respecto lo demuestran (Prado, 2008; Wanderley, 2009)4. El crecimiento y expansión de las ciudades, como en muchos casos en toda Latinoamérica, refleja con claridad aquello que Carrión denominó «la ausencia de política urbana como política urbana.

Para entender las ciudades bolivianas hay que entender cómo se urbanizaron y para comprender cómo se urbanizó el país hay que entender el curso que tomaron las migraciones (Pereira Morató: 2009; Sandoval, 1988).

Como sostienen Saravia \& Sandoval (1991) el proceso particular de urbanización de la ciudad de La Paz ${ }^{5}$ está relacionado con la migración principalmente del campo a la ciudad, fruto de la desorganización de la sociedad rural y de las expectativas que ofrece la ciudad. La incipiente industrialización no logró absorber a la población sobrante y esto se tradujo en la ubicación de vastas poblaciones en áreas periféricas con poco acceso a los componentes del consumo colectivo. Esto naturalmente trajo aparejado el respectivo deterioro de su nivel de vida (Saravia \& Sandoval, 1991: 26).

Siguiendo a Pereira Morató la formación de las ciudades bolivianas es resultado de tres factores: a) los cambios en la movilidad espacial causada por los flujos poblacionales (migración) del campo a la ciudad, b) la migración de origen urbano con destino urbano y c) el crecimiento vegetativo de la población. Otros aspectos

4 La única política urbanística específica que se desarrolló en el país es la Ley de Reforma Urbana de 1954.

5 La Paz es sede del gobierno boliviano y de los poderes Legislativo y Ejecutivo. Junto a la ciudad de El Alto forma el núcleo urbano más grande y poblado del país (el censo poblacional de 2012 reportó 1613457 habitantes en este área metropolitana). La ciudad está asentada a las riberas del río Choqueyapu, que la cruza de norte a sur, y de pequeños ríos que nacen en las laderas altas y depositan sus aguas a lo largo del trayecto del Choqueyapu, que desemboca hacia el norte del país. 
importantes son la acelerada intensidad promedio de crecimiento (3,62\% anual) y, a diferencia de las ciudades de países industrializados que se urbanizan porque requieren fuerza de trabajo concentrada, en Bolivia la urbanización respondió más bien al crecimiento de un mercado de consumo y no a un sistema productivo industrial (2009: 97-110)6.

Fruto de una embrionaria industrialización, nacida del empuje proporcionado por el endeudamiento externo en las décadas de los años 1970, el crecimiento acelerado de la ciudad de La Paz produjo una fragmentación social del espacio urbano que dio lugar además a una división étnico-social. En palabras de Calderón, citado por Saravia, se trató de «nuevas relaciones sociales y culturales, en las que no solo sobresalen los desajustes entre crecimientos poblacionales y los niveles productivos de las sociedades dependientes, y las inconsistencias relativas al crecimiento del sector terciario de la economía, sino, sobre todo, las contradicciones entre estructura productiva y los niveles de reproducción social de la población, que han generado fuertes procesos de diferenciación y empobrecimiento social» (1991: 25-26).

\section{EL PROCESO DE URBANIZACIÓN DE LA CIUDAD DE EL ALTO}

La ciudad de El Alto nació como una prolongación de La Paz. De acuerdo a Pereira, en la década de 1950 empezó a poblarse discretamente su periferia anterior, en la década de 1960 este poblamiento aumentó profusamente recibiendo la migración que se dirigía a La Paz, en los años 1970 los migrantes se ubicaron francamente en El Alto, ya no solamente como zona periférica de La Paz, y a partir de los años 1980 comenzó a crecer en todas las direcciones, pero siguiendo principalmente los ejes de transporte hacia Oruro (parte del circuito comercial que vincula al país con el puerto de Arica), hacia el Lago Titicaca (zona productiva de abastecimiento interno y de contacto comercial con el Perú) y hacia Vicha (zona industrial). Cuando fue declarada como ciudad diferenciada de La Paz (1985), el 63\% de sus pobladores no había nacido en El Alto (PNUD, 2005); en 2001 el 70\% de la población había nacido allí.

Según los datos del Censo de Población 2012, El Alto es la segunda urbe que más habitantes ha ganado en el país en cifras absolutas: de 649958 censados en 2001 ha pasado a tener 848840 en 2012, y se ubica por encima de La Paz. La sede de gobierno ha quedado detrás con 764617 habitantes, 84223 menos que El Alto.

Su rasgo central es haberse desarrollado en la lógica del abandono y la postergación (Sandóval \& Sostres, Guaygua, 2011), creando y recreando un patrón de pobreza por falta de atención a las necesidades básicas de su población. Sin servicios

6 A esta característica del proceso de urbanización boliviano Pereira (2009) denomina exógena y cita otras: dice además que es centrífuga (del centro a la periferia), lo que da lugar a la tugurización, policéntrica (tanto La Paz como El Alto tienen por lo menos cuatro grandes núcleos), metropolitana (las ciudades principales forman un conurbano con las ciudades aledañas) y no macrocefálica (a diferencia de Lima, Santiago, Buenos Aires, etc.). 
ni equipamiento, los alteños han desarrollado una existencia basada en la precariedad, lo que les ha llevado a organizarse para demandar atención de los gobernantes en varias formas de agrupación: «juntas vecinales, clubes de madres, organizaciones de educación popular, de producción, etc. En la mayoría de estas organizaciones, la pobreza aparece como el principal referente de identidad social» (Sandóval \& Sostres, 1989).

Precisamente, la creación de El Alto como ciudad tiene como antecedente la constitución de diversas organizaciones vecinales, la primera de ellas en 1945. En 1947 habían ya media docena de juntas vecinales y diez años después, en 1957, surgió el Consejo Central de Vecinos de El Alto que impulsó su reconocimiento como capital de sección municipal. En 1970 se creó la Subalcaldía de El Alto y en 1988, mediante ley, fue elevada al rango de ciudad7.

Es una idea común que la ciudad de El Alto ha servido principalmente de establecimiento para migrantes del resto del país, en especial recién llegados de las áreas rurales en busca de una oportunidad en «la ciudad». La encuesta censal de 2012 confirmó que la mayoría de los migrantes provienen de los sectores rurales de los departamentos de La Paz, Oruro y Potosí, aunque también recibe migrantes extranjeros, principalmente del sur del Perú, de los que se tienen estimaciones pero no registros oficiales. Debido a este factor migratorio, la ciudad de El Alto puede considerarse una urbe moderna, de edificaciones nuevas, algunas de las cuales reflejan mucha pobreza y otras mucha riqueza.

\section{LA PRODUCCIÓN DE LA VIVIENDA}

La situación de la vivienda en Bolivia no es distinta a la del resto de Latinoamérica, como sostiene Abramo (2011), en la construcción de las ciudades impera no solo la lógica del Estado y la del mercado sino también la lógica de la necesidad8, que es la que construye las ciudades populares latinoamericanas a través del ciclo ocupación-autoconstrucción-autourbanización-consolidación de los asentamientos informales.

7 En 1945 se formaron las primeras juntas de vecinos, se establecieron los barrios Villa Bolívar y La Ceja y más tarde una segunda junta vecinal en Villa Dolores, zona de mucha influencia todavía hoy. En 1947 se organizaron las juntas vecinales de Alto Lima, Villa Ballivián y 16 de Julio. En 1957 surgió el Consejo Central de Vecinos de El Alto. En 1970, se creó la Subalcaldía de El Alto de La Paz y el 6 de marzo de 1985 el Congreso Nacional sancionó la Ley 728 que creó la cuarta sección municipal de la provincia Murillo del departamento de La Paz cuya capital se nombraba a El Alto. Por fin, la Ley 1014, del 26 de septiembre de 1988, elevó a esta capital de sección municipal al rango de ciudad.

8 La hipótesis de Abramo es que el mercado, con su hegemonía sobre las decisiones de uso del suelo, produce una estructura «híbrida» de ciudad: en parte tomando los modelos tradicionales de la ciudad moderna («modelo mediterráneo» o «continental», compacto y con el uso del suelo intensivo) y en parte del modelo anglosajón (ciudad difusa, con uso de suelo extensivo y baja densidad). De esta manera la forma compacta y a la vez difusa produce una ciudad 'confusa' y esta estructura confusa dificulta la puesta en marcha de políticas urbanas más equitativas en términos socio-espaciales. Se trata de un círculo perverso: la compactación alimenta la difusión y esta fomenta la compactación. 
El acceso a la vivienda y al hábitat adecuado para amplios sectores de la población boliviana es una de las deudas sociales pendientes. Los esfuerzos estatales para lograr el acceso a la vivienda han sido exiguos y desacertados (Arébalo et al., 2011: 128-129). Los programas de vivienda tradicionalmente estuvieron dirigidos a los asalariados — sector que no representa al contingente más empobrecidoposiblemente porque el Estado requería reproducir la fuerza de trabajo en actividades estratégicas o porque el gobierno de turno necesitaba contener la presión social. Así, en general, no se desarrollaron programas de vivienda en función de las necesidades, demandas y particularidades de la población, la «resolución de la problemática de la vivienda» no fue una respuesta institucional a los derechos básicos de las personas sino un mecanismo de cooptación de organizaciones sociales o una manera de lograr rédito político.

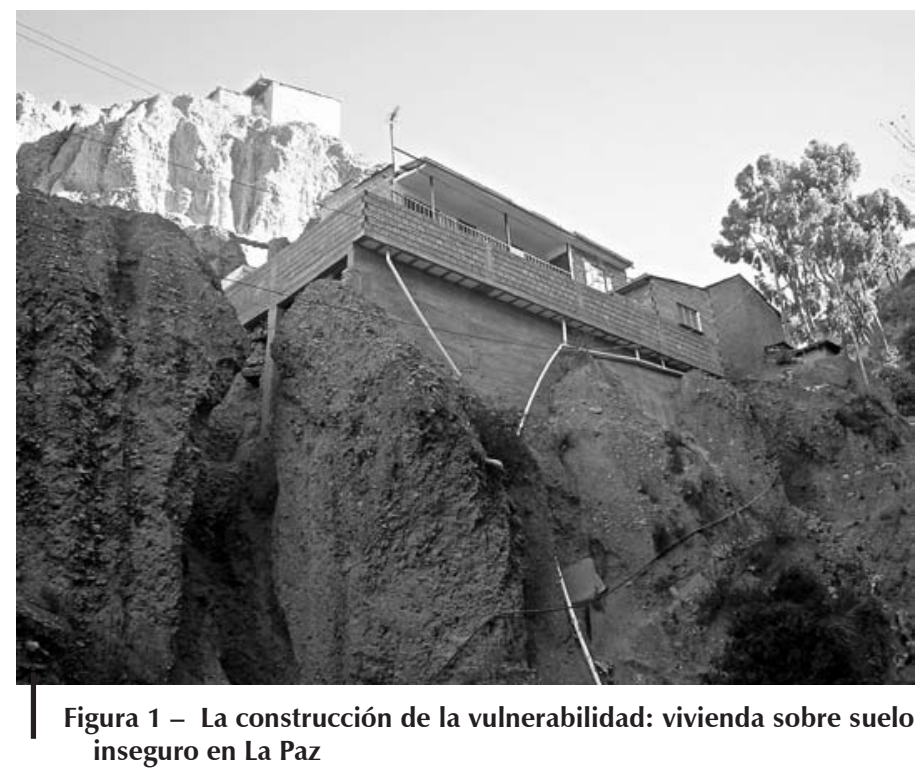

Foto: Fabien Nathan, 2004

Con todo, el $63 \%$ de la población cuenta con vivienda propia, el déficit es cualitativo: la vivienda es inadecuada. El déficit por carencia (cuantitativo) y por mala calidad (cualitativo) impactan particularmente en sectores de la población social, política y económicamente más vulnerables (Arébalo, 2011). Nuevamente, como en toda América Latina, los procesos de Producción Social del Hábitat en Bolivia nacen de la necesidad de vivienda y se apoyan en la capacidad organizativa de la gente, de modo que el $80 \%$ de las viviendas son autoproducidas, lo que está directamente relacionado con la calidad no solo de la vivienda sino del hábitat y con la construcción de vulnerabilidad. 


\section{LA VULNERABILIDAD URBANA ${ }^{9}$ EN ESTAS CIUDADES}

Las ciudades de El Alto y La Paz forman juntas la primera aglomeración urbana más grande de Bolivia con 1613457 habitantes 10 y poseen una fuerte relación de interdependencia tanto en lo productivo, comercial como social. Muchos alteños venden sus productos en los mercados de La Paz y una gran cantidad de empresas paceñas instalan sus fábricas en El Alto11, razón por la cual contratan empleados y mano de obra alteña; por otra parte, muchas familias extendidas tienen doble residencia, una casa en La Paz cerca de su fuente laboral y otra en El Alto, cerca de su comunidad de origen; muchas personas que viven en La Paz compran su alimento, vestido y otros objetos en las ferias de El Alto, y muchos residentes alteños tienen su fuente de trabajo en La Paz. Los límites formales entre estos dos municipios son invisibles a su dinámica cotidiana.

Aunque existe una verdadera interfaz ${ }^{12}$ entre La Paz y El Alto, y ambas ciudades coinciden en la problemática que deriva de su acelerado crecimiento y proceso de urbanización, los factores y causas de su vulnerabilidad son muy distintos.

Privilegiada por una continuidad institucional de más de una década en el gobierno de su municipio, la vulnerabilidad de la ciudad de La Paz es sobre todo física debido a sus características geomorfológicas. Al contrario, asentada en una amplísima meseta —no libre de sismos y deslizamientos de tierras por sus pequeños ríos subterráneos- en la ciudad de El Alto las amenazas naturales son las inundaciones (Sandoval \& Sostres, 1989), pero su principal vulnerabilidad es el elevadísimo nivel de conflictividad y la falta de gobernabilidad que comprometen su desarrollo económico y social, a lo que podría aplicarse la noción de vulnerabilidad «de fondo» que D'Ercole et al. (2009 b) describen como resultante de procesos socio-históricos de largo plazo.

9 Utilizamos el término vulnerabilidad urbana en el sentido que propone Blaikie (cit. por D'Ercole et al., 2009b): como la dificultad de las ciudades para anticipar y enfrentar fenómenos físicos o antrópicos (sismos, inundaciones, deslizamientos de tierra, incendios, explosiones o contaminaciones, etc.) capaces de causar daño, pero también para enfrentarlos y superarlos después de su ocurrencia; y como la capacidad de las ciudades y sus habitantes en fomentar estos fenómenos o al menos de multiplicar sus efectos. Por su parte, la Dirección Especial de Gestión Integral de Riesgos (DEGIR), del Gobierno Autónomo Municipal de La Paz, define vulnerabilidad como el «factor interno de riesgo de un sujeto, objeto o sistema, expuesto a una amenaza, que corresponde a su disposición intrínseca a ser dañado. Es el grado de fortaleza que presenta el sujeto, objeto o sistema ante un determinado evento; por lo que si esta susceptibilidad de ser dañado es grande se dice que tiene una alta vulnerabilidad y en caso contrario, que tiene una baja vulnerabilidad» (GAMLP, 2011: 83).

10 En: http://www.la-razon.com/sociedad/La_Paz-poblacion-El_Alto-Santa_Cruz_0_1883211728.html (revisado el 31/1/2014).

11 La actividad comercial minorista en El Alto es muy grande, hay alrededor de 5600 pymes, fábricas y plantas de procesamiento de hidrocarburos; es lugar de exportación de los recursos minerales del país y de materia prima procesada.

12 En informática es la «superficie de contacto», la conexión física y funcional entre dos sistemas o dispositivos dando una comunicación entre distintos niveles. La interfaz como espacio es el lugar de la interacción, el espacio donde se desarrollan los intercambios. 
A pesar de ello, como previenen algunos estudiosos, al estar estas ciudades a cerca de 4000 metros sobre el nivel del mar «los eventos son en su mayoría de pequeña y mediana magnitud y principalmente de carácter hidromorfológico [...] las preocupaciones están todavía muy focalizadas en los riesgos de origen natural aun cuando los fenómenos de origen antrópico son cada vez más evidentes».

\section{1. La Paz}

La ciudad de La Paz presenta condiciones naturales muy complejas por su configuración geológica, geotécnica y por su topografía de alta pendiente. Ubicada en una cuenca drenada por cientos de ríos y riachuelos, sus flancos muestran erosión regresiva y sus lechos acumulación de materiales.

Son muchos los factores de vulnerabilidad en esta ciudad —baja gestión ambiental de los desechos sólidos, construcciones precarias (casas con muros de adobe, techos en calamina y pisos de tierra); invasión de áreas susceptibles a inundaciones; pobreza y analfabetismo; déficit en la provisión de servicios de energía eléctrica, alcantarillado y agua potable- pero sin duda la precariedad del suelo es la principal: el $74 \%$ de la población asienta sus viviendas en suelo inestable13.

Siendo los eventos climáticos y sobre todo hidromorfológicos los más frecuentes (D’Ercole et al., 2009a: 449), deslizamientos, derrumbes, mazamorras y flujos de barro recaen en especial en los barrios periféricos desarrollados en pendientes

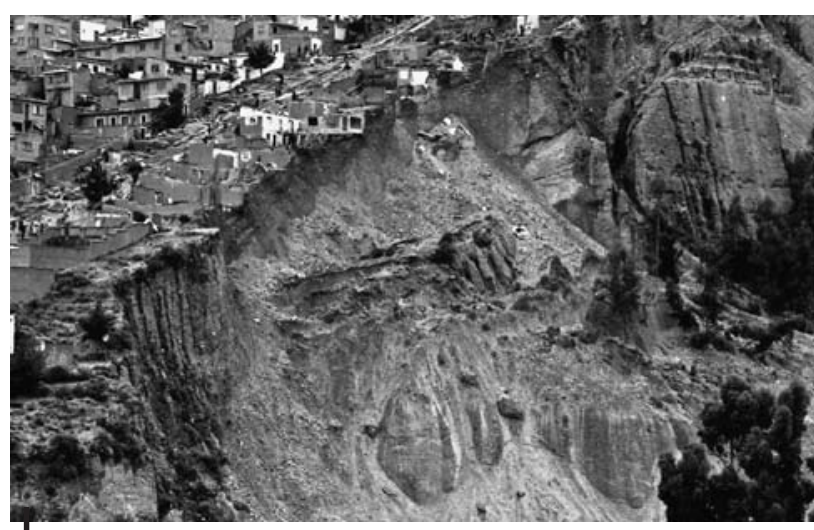

Figura 2 - Deslizamiento en la zona de Alto Tacagua, La Paz

Foto: Luis Salamanca inestables y resultan la mayor amenaza por la presión del agua fluvial que satura estos suelos que de acuerdo a los registros históricosen su gran mayoría ceden en caída lenta, razón por la cual los daños son principalmente materiales, de la infraestructura urbana y por eso son considerados eventos menores en número de muertos, en un contexto de análisis comparativo ${ }^{14}$.

Estos eventos adversos, generalmente reconocidos como fenómenos naturales externos, se incrementan por procesos de la actividad humana

13 En 1977 se encargó de la elaboración de un mapa de constructibilidad que determinó que el 62\% de los terrenos no tenían condiciones favorables para la construcción, y en 1999 un nuevo estudio dio como resultado que $74 \%$ del suelo no era apto para construir (Programa de Gestión Integral del Riesgo del GAMLP).

14 El trabajo de D'Ercole et al. (2009a) ampliamente citado hace un balance de los desastres ocurridos en el área andina: Bolivia, Perú y Ecuador. 
como el crecimiento urbano desordenado y la construción en zonas no aptas para este fin, agravando las condiciones de inestabilidad geológica, socavando taludes empinados, rellenando quebradas sin planificación técnica y en otros casos por infiltración de aguas servidas. Como sostienen D’Ercole et al.:

resulta difícil distinguir los fenómenos de origen natural de los fenómenos antrópicos en función de un encadenamiento complejo de efectos, típico de los medios urbanos. Si bien los orígenes son a veces naturales, los efectos son siempre antrópicos (2009a: 464).

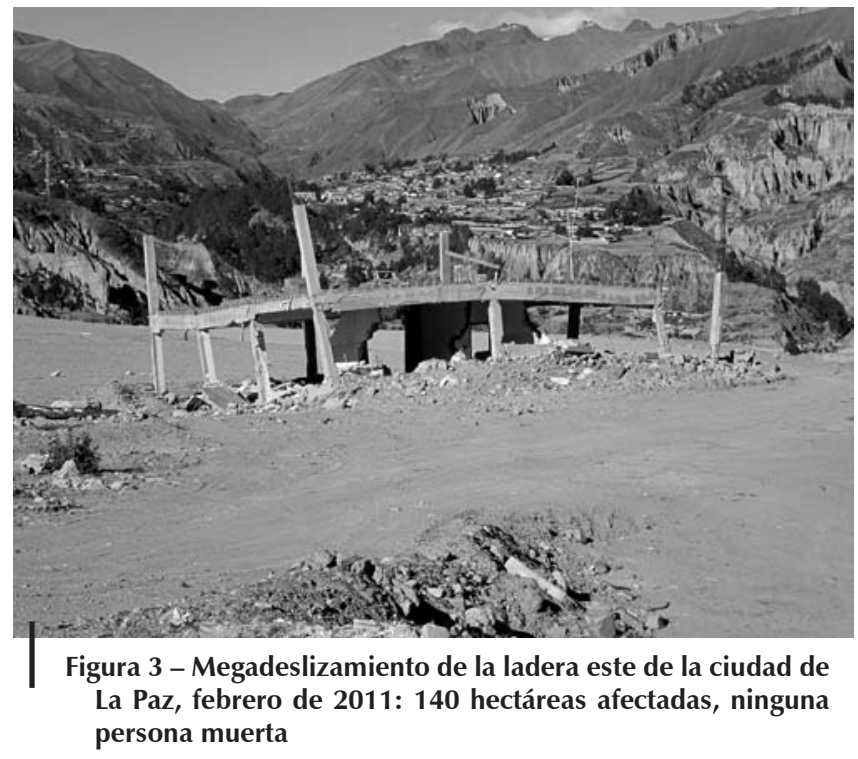

Foto: P. Urquieta

\section{2. El Alto}

A pesar de la ausencia total de vegetación (Sandóval \& Sostres, 1989) y las condiciones de semiaridez (Hardy, 2009b), la constitución física de la ciudad de El Alto es un poco más favorable que la de La Paz, razón por la cual los eventos de desastre de origen natural y antrópico son comparativamente menos numerosos y están relacionados principalmente con el daño de la producción agrícola ocasionado por las temperaturas muy bajas, tormentas de viento, inundaciones, deslizamientos de tierra, y violentas precipitaciones de nieve, lluvia y granizo (Montes de Oca, cit. por Hardy, 2009b). Y aunque las amenazas de origen natural en esta ciudad están identificadas con precisión, los análisis demuestran que existen procesos formados por el hombre (Hardy, 2009b: 880-885) —fuerte crecimiento demográfico y espacial en un territorio con poco control-, que al mismo tiempo que constituyen una coerción para las actividades humanas, también son resultado de ellas. 


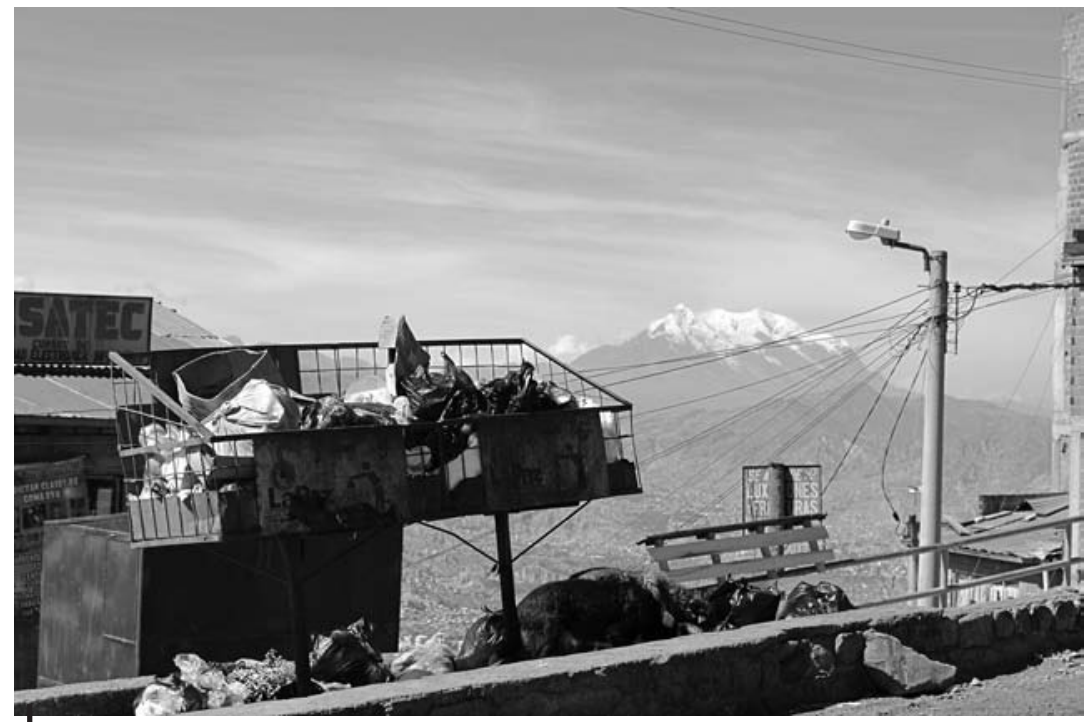

Figura 4 - Deficiencias en la gestión de residuos sólidos, falta de normativas en algunos aspectos e incumplimiento de normas que existen son parte de las dificultades que tiene la ciudad de El Alto en su gestión

Foto: Manuela Paz, 2014

A todo lo descrito es posible añadir otros dos factores de riesgo: el elevado nivel de conflictividad y la inseguridad urbana. La movilización social (Guaygua, 2011) para lograr atención a las necesidades básicas de su población (carente de servicios básicos y de infraestructura urbana) con mucha frecuencia provoca pugnas entre dirigentes de organizaciones vecinales, gremiales, juntas escolares, etc. que rebasan los mecanismos institucionales y derivan en conflictos violentos. El Alto ocupa el primer lugar en el país en cuanto a violencia contra las personas o violencia interpersonal 15 .

En la práctica, la forma de gobierno de la municipalidad de El Alto es no convencional. La gestión se lleva a cabo en coordinación con los representantes de estas organizaciones. La ventaja de este empoderamiento social radica en que las demandas se canalizan de forma directa, pero a la vez existe el riesgo de que al participar las dirigencias en la vida política de la ciudad, también de manera directa, engrosen las filas de la corrupción y que los recursos económicos de la ciudad se utilicen en acciones más vinculadas a intereses particulares y de grupo que a los del conjunto de la población.

15 La primera encuesta de victimización, prácticas y percepción sobre violencia y delito —realizada en enero de 2012 en La Paz, El Alto, Cochabamba y Santa Cruz por el Viceministerio de Seguridad Ciudadana - muestra que el robo y el hurto son proporcionalmente mayores en Cochabamba y El Alto. Boletín del Observatorio Nacional de Seguridad Ciudadana, año 1, número 1. 


\section{CORRESPONSABILIDAD ENTRE ESTADO Y SOCIEDAD}

A pesar de la importante interdependencia descrita persiste una mirada dicotómica entre las municipalidades de La Paz y de El Alto, basada en aspectos históricos, económicos y sobre todo sociales, que «no facilita la construcción de una visión de conjunto de los riesgos y de sus efectos» sobre este territorio común ( $\mathrm{D}^{\prime}$ Ercole et al., 2009a: 463).

Frente a condiciones estructurales tan complejas cabe preguntarse hasta qué punto son estas modificables a través de políticas públicas enfocadas simplemente en acciones de educación, capacitación y sensibilización. Muchos estudios revelan que las personas conviven con el riesgo, que los vecinos conocen los problemas antes de habitar o construir pero lo hacen porque esas viviendas o terrenos son lo único accesible a sus posibilidades económicas y «una vez que comienzan a rajarse las paredes o quebrarse el suelo, utilizan sus conocimientos culturales tratando de tapar la 'evidencia' curando las paredes y taconeando los pisos, y en muchos casos cuando es época de lluvias, no duermen por las noches, [por temor a que] les ocurra algo malo» (Salamanca, 2009: 212).

Es importante crear conocimiento, identificar las amenazas, analizar los elementos o componentes del riesgo y lograr el reconocimiento de las diversas formas de vulnerabilidad, pero todo ello debe analizarse en un marco cotidiano, cercano a la realidad y en el contexto específico de los grupos vulnerables. El uso del espacio, la forma de ocupación del suelo, requiere una mirada multidisciplinaria que reconozca y distinga los hábitos y comportamientos que responden a condiciones materiales concretas y en muchos casos estructurales, y que por lo tanto no pueden ser simplemente «combatibles»16, de aquellos que son fruto de la falta de información, malas prácticas o conocimientos falsos o erróneos que se practican por emulación ${ }^{17}$.

La mayoría de los países cuenta con políticas de prevención de desastres y gestión de riesgos en general, pero estas están centradas en la necesidad de intervención y control de la emergencia18. Aunque tiene como principio «resguardar el bienestar común [...] procurando reducir la situación de vulnerabilidad y el retorno a la normalidad»19, la Ley Municipal de Gestión Integral de Riesgos de la ciudad de La

16 « ... sí, nosotros hemos comprado en un lugar pendiente, en un lugar malo el terreno... pero cómo podemos acceder a este otro lugar que es más costoso... Tal vez por tener como siempre alguien dice en su verbo de alguien dice [sic] 'tener casa hasta en la punta del cerro pero que sea tuyo, que sea propio'. Tal vez por azares de la vida ha logrado conseguir esto, y no podemos acceder a este otro lugar, porque es un poco más costoso...» (vecino de Germán Jordán, en Salamanca, 2009).

17 Desconocimiento de la presencia de aguas subterráneas; la sobrecarga que ocasionan las edificaciones de varios pisos y el riesgo que constituye la construcción fuera de norma; la mala disposición de aguas servidas y utilización de pozos ciegos.

${ }^{18}$ Estimación del riesgo, monitoreo, instrumentos; declaratoria y evaluación de la emergencia, evacuación, demolición, reposición de servicios, etc.

19 Ley Municipal Autonómica № 005/2010. Gobierno Autónomo Municipal de La Paz. Capítulo II. Principios, definiciones y siglas. Artículo $4^{\circ}$.- (Principios) Los principios que rigen la presente ley 
Paz descuida la etapa de reconstrucción, de la que hay apenas dos referencias: un artículo relacionado con la rehabilitación del área afectada y su reordenamiento territorial con el objetivo de mitigar el riesgo de un futuro evento, y otro referido a la inversión para damnificados, previendo recursos para programas de construcción de viviendas.

Una vez que ocurre el desastre la gestión institucional del riesgo debe poner el énfasis en apoyar la construcción de resiliencia 20 en las comunidades. En esta ley no existe alusión - por ejemplo - a que las acciones y políticas en la etapa de reconstrucción deben tomar en cuenta la importancia de la reconstitución del tejido social.

Es conocido el poder de las poblaciones de La Paz y El Alto para organizarse y resolver la atención a sus problemas y necesidades, y es igualmente reconocida su capacidad resiliente, pero se sabe también que es una sociedad todavía desinformada en muchos aspectos y que persisten prácticas cotidianas que agravan la precariedad de sus viviendas y su hábitat y que, por lo tanto, aumentan el riesgo.

La participación debe cualificarse a través de más conocimiento, mejor interacción, construcción de confianza y desde un enfoque de corresponsabilidad.

Corresponsabilidad significa que la sociedad y el Estado están dispuestos a asumir el desafío de la cogestión, entendiendo los nuevos alcances que este modelo impone a los roles tradicionales: los ciudadanos y ciudadanas dejan de ver al Estado como el único operador del cambio y el Estado altera su rol omnímodo y paternalista. Corresponsabilidad también implica intervención en decisiones; supone descentralización, democratización del poder y de los mecanismos de acceder a él; exige capacidad de interlocución, negociación y concertación de todos los actores involucrados.

La cogestión del riesgo consiste en un Estado más social, mejor informado con conocimiento, comprensión y sensibilidad respecto del espacio como una construcción social; y una sociedad más informada, más abierta a gestionar el riesgo con mayor conocimiento técnico y que canaliza sus fortalezas hacia la construcción compartida de la ciudad.

son: a) Interés colectivo: La gestión integral de riesgos de desastres está enfocada a resguardar el bienestar común con la preservación e integridad física de los habitantes y la implementación de acciones a favor de la colectividad, procurando reducir la situación de vulnerabilidad y el retorno a la normalidad.

20 En términos amplios la resiliencia es la capacidad que tiene una persona —o un grupo de personasde recuperarse luego de enfrentar una adversidad, para seguir proyectando su futuro. 


\section{Referencias citadas}

ABRAMO, P., 2011 - La ciudad confusa. La mano inoxidable del mercado y la producción de la estructura urbana en las grandes metrópolis latinoamericanas. In: La producción de las ciudades latinoamericanas: mercado inmobiliario y estructura urbana (P. Abramo, ed.): 337-380; Quito: Organización Latinoamericana y del Caribe de Centros Históricos (OLACCHI), Municipio del Distrito Metropolitano de Quito (MDMQ). Colección Textos Urbanos, 9.

ARÉBALO, M., BAZOBERRY, G. \& LANDAETA, G., 2011 - La producción social del hábitat en Bolivia. In: El camino posible. Producción Social del Hábitat en América Latina: 127-147; Montevideo: Centro Cooperativo Sueco-TRILCE.

BOLIVIA, 2010 - Ley Municipal Autónoma de Gestión Integral de Riesgos de Desastres № 005/2010 del 29 de diciembre de 2010. Gobierno Autónomo Municipal de La Paz.

CARRIÓN, F., 1988 - La urbanización andina: notas sobre el estado del conocimiento. In: Investigación Urbana en el Área Andina (F. Carrión, ed.): 11-44; Quito, CuidadInstituto Francés de Estudios Andinos (IFEA).

D'ERCOLE, R., HARDY, S. \& ROBERT, J., 2009a - Balance de los accidentes y desastres ocurridos en La Paz, Lima y Quito (1970-2007). Bulletin de l'Institut Français d'Études Andines, 38 (3): 433-465.

D'ERCOLE, R., HARDY, S., METZGER, P. \& ROBERT, J., 2009b - Vulnerabilidades urbanas en los países andinos. Introducción general. Bulletin de I'Institut Français d'Études Andines, 38 (3): 401-410.

FORO PERMANENTE DE LA VIVIENDA (FOPEVI), 2009 - Propuesta de Política Estatal de Vivienda; La Paz: Foro Permanente de Vivienda, Red Hábitat.

GOBIERNO AUTÓNOMO MUNICIPAL DE LA PAZ, 2011 - Memoria del deslizamiento del complejo Pampahasi-Callapa del 26 de febrero de 2011; La Paz: Anakainoó.

GUAYGUA, G., 2011 - Identidad colectiva y movilidad social en la ciudad de El Alto. En: Nuevas identidades urbanas. Tres miradas desde la cultura y la desigualdad (G. Guaygua, C. Peña \& A. Waldmann, eds.): 237-318; La Paz: Programa de Naciones Unidas para el Desarrollo (PNUD). Cuaderno de Futuro 27. Informe sobre Desarrollo Humano.

HARDY, S., 2009a - Granizada e inundación del 19 de febrero de 2002. Un modelo de crisis para la aglomeración de La Paz. Bulletin de I'Institut Français d'Études Andines, 38 (3): 501-514.

HARDY, S., 2009b - Evaluación y gestión de la vulnerabilidad de los distritos 5 y 6 del municipio de El Alto. Bulletin de I'Institut Français d'Études Andines, 38 (3): 873892.

HARVEY, D., 2008 - El derecho a la ciudad. New Left Review, 53, noviembre-diciembre. Versión electrónica disponible en http://newleftreview.es/53

HIERNAUX, D. \& LINDÓN, A.,1996 - El concepto de espacio y el análisis regional. Secuencias, núm. 25 de enero a abril: 89-110; México: Instituto Mora.

LEFEBVRE, H., 1974 - La producción del espacio. Papers. Revista de Sociología, 3: 219-229.

PARK, R., 1999 - La ciudad y otros ensayos de ecología urbana, 148 pp.; Barcelona: Ediciones del Serbal.

MAZUREK, H., 2006 - Espacio y territorio. Instrumentos metodológicos de investigación social, 206 pp.; La Paz: PIEB, IRD. 
PEREIRA MORATÓ, R., 2009 - Las ciudades bolivianas, ¿cómo entenderlas? Migración y urbanización. In: Estudios Urbanos en la encrucijada de la interdisciplinariedad (F. Wanderley, ed.): 95-117; La Paz: CIDES-UMSA Colección 25 Aniversario, EPFL/ NCCR North-South.

PRADO SALMÓN, F., 2008 - El descuidado tema urbano en la Bolivia de hoy. Tinkazos, 25: 11-85, noviembre. Revista Boliviana de Ciencias Sociales del Programa de Investigación Estratégica en Bolivia (PIEB).

PROGRAMA DE NACIONES UNIDAS PARA EL DESARROLLO (PNUD), 2005 - Informe sobre Desarrollo Humano 2005. La economía más allá del gas; La Paz.

SALAMANCA, L., 2009 - Estudio de resiliencia en desastres naturales en seis barrios de la ciudad de La Paz, Bolivia. In: La gestión del riesgo urbano en América Latina: recopilación de artículos. Plataforma Temática de Riesgo Urbano. EIRD: 206-222. Versión electrónica disponible en http://www.eird.org/plataforma-tematica-riesgourbano/recopilacion-de-articulos/luis-salamanca.pdf

SANDOVAL, G., 1988 - Investigación urbana en Bolivia. In: Investigación Urbana en el Área Andina (F. Carrión, ed.): 47-62; Quito: Cuidad-IFEA.

SANDOVAL, G. \& SOSTRES, F., 1989 - La ciudad prometida. Pobladores y organizaciones sociales In: El Alto, 230 pp.; La Paz: ILDIS-SYSTEMA.

SARAVIA, J. \& SANDOVAL, G., 1991 - Observando el complejo mundo urbano. In: Jach'a uru: ¿La esperanza de un pueblo?: 25-42; La Paz: ILDIS/CEP.

URQUIETA, P., 2010 - Introducción. In: Ciudades en transformación: disputas por el espacio, apropiación de la ciudad y prácticas de ciudadanía (P. Urquieta, ed.): 1328; La Paz: CIDES-UMSA- EPFL/NCCR North-South - Oxfam - Plural.

VICEMINISTERIO DE SEGURIDAD CIUDADANA, BOLIVIA, 2012 - La primera encuesta de victimización, prácticas y percepción sobre violencia y delito, Boletín del Observatorio Nacional de Seguridad Ciudadana, año 1, número 1.

VILLAFAÑEZ, E. A., 2011 - Entre la geografía y la arqueología: el espacio como objeto y representación. Revista Geográfica Norte Grande, 50, dic.: 135-150; Santiago. Versión electrónica disponible en http://www.scielo.cl/scielo.php?script=sci_ arttext\&pid $=$ S071834022011000300008\&lng =es\&nrm $=$ iso

WANDERLEY, F., 2009 - Introducción: Los estudios urbanos en la encrucijada de la interdisciplinariedad. In: Estudios Urbanos en la encrucijada de la interdisciplinariedad (F. Wanderley, ed.): 13-23; La Paz: CIDES-UMSA Colección 25 Aniversario, EPFL/ NCCR North-South - Plural. 